\title{
DNA Separation Chips \\ Using Temporally Asymmetric Ratchet Effect in Nonuniform Electric Fields
}

\author{
Soyeon Yi and Young-Ho Cho* \\ Digital Nanolocomotion Center \\ Department of Bio \& Brain Engineering, \\ Korea Advanced Institute of Science and Technology \\ 373-1 Guseong-dong, Yuseong-gu, Daejeon 305-701, Republic of Korea
}

(Received September 3, 2008; accepted December 24, 2008)

Key words: bio-separators, electrophoresis, DNA ratcheting chips, nonuniformly distributed asymmetric electric fields

We present DNA separation chips using the temporally asymmetric ratchet effect in nonuniform electric fields. The present separation chip redistributes DNA within a specific area in asymmetrically switched nonuniform electric fields based on the size- and field-dependent nonlinearities of DNA drift velocity. Compared with the conventional electrophoresis chips, the present separation chip can be easily integrated into automated DNA analysis systems because of its simple structure and starting-point-independent DNA separation. On the basis of the drift velocity of three different DNA molecules $(11.1,15.6$ and $48.5 \mathrm{kbp})$, we extract the asymmetric alternating electric field conditions $\left(E_{1}=4 E_{2}\right.$ and $\left.23 T_{1}=3 T_{2}\right)$, where Phagemid DNA (15.6 kbp) shows zero net velocity while EM3 DNA (11.1 kbp) and $\lambda$ DNA (48.5 kbp) migrate to the $-x$ and $+x$ directions, respectively. The present chip is composed of a tapered channel to generate nonuniform electric fields, a DNA loading slit, and a pair of electrodes to apply the electric field. We focus on the design of the DNA separation chips to identify the nonlinearity of DNA drift velocity using three different DNA molecules in the chips. It is demonstrated that different sizes of DNA show different net migration velocities under the nonuniformly distributed asymmetric alternating electric fields. Phagemid DNA moved to its own specific location, $-1.5 \mathrm{~mm}$ from the starting point ( $+2 \mathrm{~mm}$ from the loading slit), then showed zero net migration velocity. Other sample DNA molecules, EM3 and $\lambda$ DNA, migrated $2.2 \mathrm{~mm}$ in the $-x$ direction and $1 \mathrm{~mm}$ in the $+x$ direction, respectively, under the alternating asymmetric electric field, toward their own specific locations where they show net zero velocity. Also discussed are the potentials of the present DNA chips for the miniaturization of DNA analysis systems and the potentials of the present DNA chips having tunable capability of the target DNA size to be separated.

${ }^{*}$ Corresponding author: e-mail: nanosys@kaist.ac.kr 


\section{Introduction}

The DNA separation device is one of the key components of micro DNA analysis systems, ${ }^{(1)}$ since efficient methods to separate and analyze the different components of a mixture of DNA molecules are of paramount importance, both for research and in biomedical applications. To meet this need, a concerted effort is being made to develop miniaturized bioanalytical devices using the microfabrication technology. As a result of these concerted efforts, several microelectrophoresis chips ${ }^{(2-6)}$ use microlithographic arrays as an artificial gel instead of gel matrix to realize a uniform sieving matrix. To improve the separation efficiency, the electric field directions ${ }^{(2,5,6)}$ or design-specific patterns and geometry of microarrays ${ }^{(4)}$ are changed as occasion demands. Compared with the conventional electrophoresis chips, the present separation chip can be easily integrated into automated DNA analysis systems because of its simple structure and starting-point-independent DNA separation.

Recently, some remarkable DNA properties, ${ }^{(7-9)}$ such as the nonlinear nature of the DNA drift velocity, have been discovered in the electric fields relevant to the physics of macromolecules and biopolymers. Interestingly, these new phenomena in the behavior of DNA in the electric fields may also be observed using the common gel electrophoresis and pulsed field gel electrophoresis (PFGE) techniques. To improve the separation capability of DNA PFGE, an asymmetric periodic electric field with a zero average value $^{(10)}$ is introduced. This electric field offers possibilities to manipulate the total drift velocity at will, such as macromolecules of different sizes can be made to move in opposite directions in PFGE. By comparing with the ratcheting of the DNA molecules in modified conventional gel electrophoresis systems, ${ }^{(10)}$ through the miniaturization of the chip, we may also possibly shorten the process time and minimize the disadvantages of the nonuniform electric fields, temperature field in the gel, and curvilinear shape of the separated fractions.

The present separation chip (Fig. 1) redistributes DNA molecules within a specific area based on the ratchet effect(11) using asymmetric alternating electric fields due to size- and field-dependent nonlinearities of the DNA drift velocity. Determination of the asymmetric alternating electric field condition has been deduced on the basis of the measured DNA migration velocities of the DNA molecules, without being limited by a zero average value. ${ }^{(10)}$ This DNA ratchet effect allows the separation chip to be automated for integration in DNA analysis systems.

The proposed separation chip is to be applied in a rapid, inexpensive restriction fingerprinting process, which is utilized in restricted fragment length polymorphism (RFLP), ${ }^{(12,13)}$ screening of embryonic stem cell clones for gene targeting ${ }^{(14)}$ or screening of transgenic animals. ${ }^{(15)}$ Thanks to the temporally asymmetric ratchet effect in this chip, DNA fragments in a wide size range could be separable in a short channel instead of long filaments that are usually used in RFLP techniques for genetic linkage studies, such as paternity testing and genetic fingerprinting. In addition, the proposed chip can be applicable to the screening process for building bacterial artificial chromosome (BAC) ${ }^{(16)}$ libraries by identifying DNA molecules of specific sizes. Since DNA molecules of specific sizes migrate to their own specific locations under asymmetric alternating electric fields, it is easy to check the existence of DNA molecules of specific sizes. 


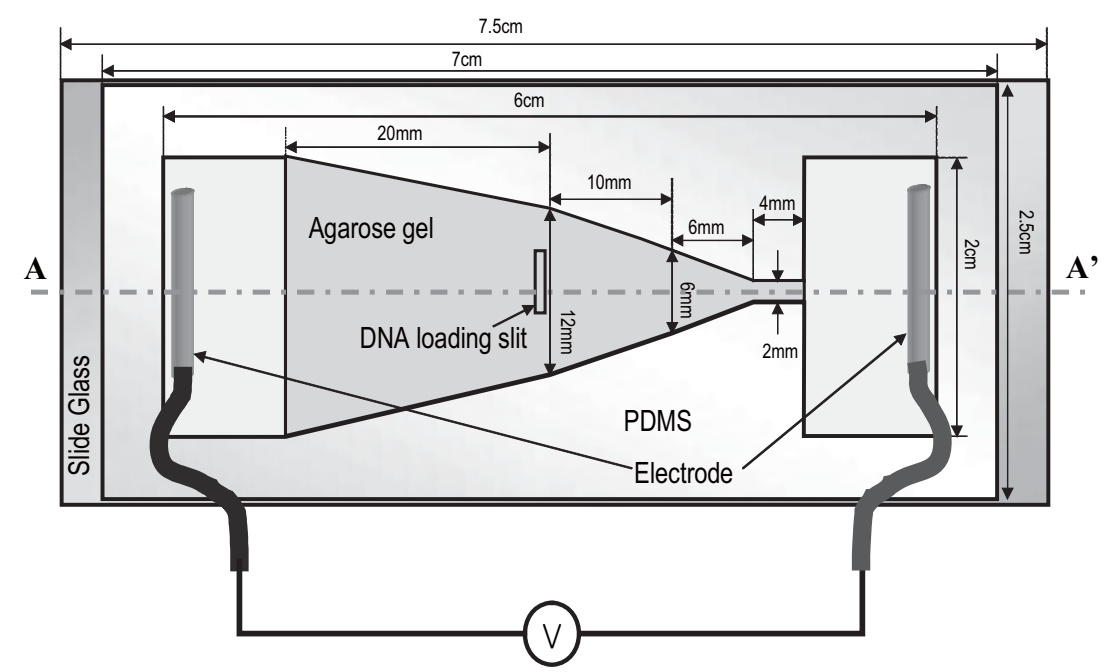

Fig. 1. Top view of the DNA separation chip with designed dimensions.

\section{DNA Separation Principle}

\subsection{Temporally asymmetric ratchet effect}

Figure 2 shows the principle of DNA ratcheting and separation using the temporally asymmetric ratchet effect due to size- and field-dependent nonlinearities of the DNA drift velocity. When a forward electric field, $E_{1}$ is applied for time, $T_{1}$, and then switched periodically to the backward field, $E_{2}$, for $T_{2}$ as shown in Fig. 2(a), in the horizontal direction of the channel, the DNA molecules in the channel (Fig. 2(b)) move forward and backward with the specific velocities depending on their molecular sizes. Given the values of $E_{1} / E_{2}$ and $T_{1} / T_{2}$, for example, the DNA of mass $M_{2}$ (Fig. 2(b)) simply oscillates at a specific location with zero net velocity, while the others of masses, $M_{1}$ and $M_{3}$ move to the $-x$ and $+x$ directions, respectively. This phenomenon is based on the drift velocity of the DNA molecules, which is not linear to the electric field intensity and to the size of the DNA molecules. Whatever is the source of this nonlinearity, we can use such a system to build a correlative ratchet that rectifies temporally biased fluctuations. ${ }^{(11)}$

\subsection{Nonuniform electric field distribution}

According to the temporally asymmetric ratchet effect, we can make a DNA molecule of a specific size oscillate at a specific location with zero net velocity. To make DNA molecules of other sizes migrate to their own specific locations with zero net velocity, we need to distribute the electric fields nonuniformly along the channel. For the nonunifrom electric field distribution, we vary the channel width (Fig. 1) so that 


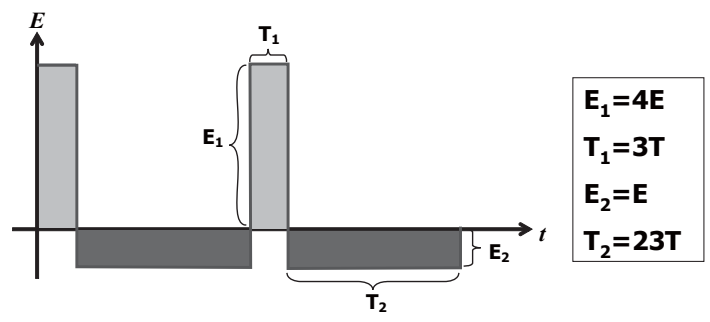

(a)

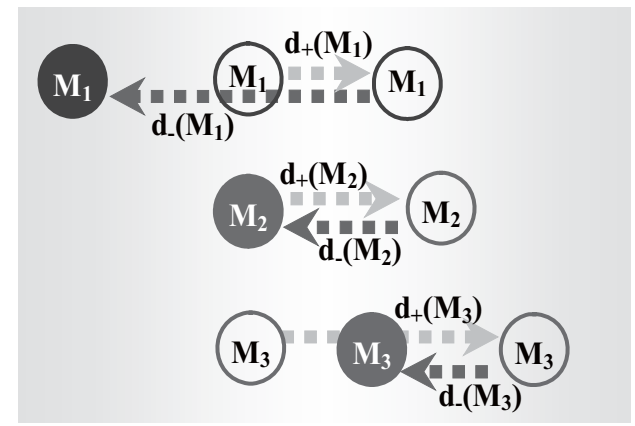

(b)

Fig. 2. Asymmetrically alternating electric fields for DNA separation: (a) asymmetrically alternating electric field and time conditions for DNA separation; (b) size-dependent DNA migration in the asymmetrically alternating electric fields of $E_{1}$ for $T_{1}$ and $-E_{2}$ for $T_{2}$.

nonuniform electric field zones can be generated in the channel filled with agarose gel. By varying the channel width, we can distribute the asymmetric alternating electric field train (Fig. 2(a)) having a gradient of the intensity along the channel, keeping the same ratio and period. Therefore, by applying the asymmetric alternating electric fields in the width-variable channel, $M_{1}$ and $M_{3}$ can also oscillate as well as $M_{2}$ at the specific location with net zero velocity. Consequently, DNA molecules of different sizes migrate with different velocities toward their own specific locations. After reaching their own specific locations, they would have a zero net velocity and then just oscillate near these locations in response to the applied asymmetric electric field train.

\section{Design and Fabrication}

To realize the DNA separation in a chip, the asymmetric alternating nonuniform electric field with intensity variation through one channel should be generated. Then, each DNA molecule can have its own specific location where the migration velocity becomes zero. To estimate the DNA migration and design the DNA separation chip using agarose gel, we measured the DNA migration velocity in agarose gel with the applied voltage. We measured the migration velocities of the three types of DNA molecules, DNA I (EM3), II (Phargemid) and III ( $\lambda$ DNA) having different sizes of 11.1, 15.6 and $48.5 \mathrm{kbp}$, respectively. In this process, we used $0.5 \mathrm{X}$ Tris-Acetate-EDTA (TAE) buffer for the DNA electrophoresis. To detect the DNA molecules after electrophoresis, the DNA molecules were stained with ethidium bromide $(\mathrm{EtBr})$ by mixing $\mathrm{EtBr}$ with the agarose gel before electrophoresis and/or dipping the agarose gel in the EtBr solution after the electrophoresis, as occasion demands. We varied the applied voltage from 5 to $240 \mathrm{~V}$ in the conventional gel electrophoresis kit to measure the drift velocity of DNA molecules according to the applied voltage with the fluorescence image of the gel and 
the process time. Through the Maxwell simulation of the gel bath of the conventional gel electrophoresis kit, we estimated the magnitude of the electric field of each applied voltage.

\subsection{Electric field condition}

On the basis of the measured DNA migration velocities in Fig. 3, we estimated the asymmetric alternating electric field condition. First, we estimated the condition that will make DNA II oscillate in the channel. When $E_{1}$ is $640 \mathrm{~V} / \mathrm{m}, E_{2}$ is $160 \mathrm{~V} / \mathrm{m}$ (Fig. 3), and $23 T_{1}$ is the same as $3 T_{2}$, DNA II shows behavior like that of $\mathrm{M}_{2}$ in Fig. 2(b), while DNA I and DNA III migrate to the $-x$ and $+x$ directions, respectively. We calculated the net migration velocities of the sample DNA molecules under the conditions of $E_{1}$ is $4 E_{2}$ and $23 T_{1}$ is $3 T_{2}$, and estimated the migration velocities of the sample DNA molecules along the channel.

\subsection{Chip design and estimation of DNA migration}

From the asymmetric alternating electric field condition and the migration velocities of the three different DNA molecules, we designed the DNA separation chip as shown in

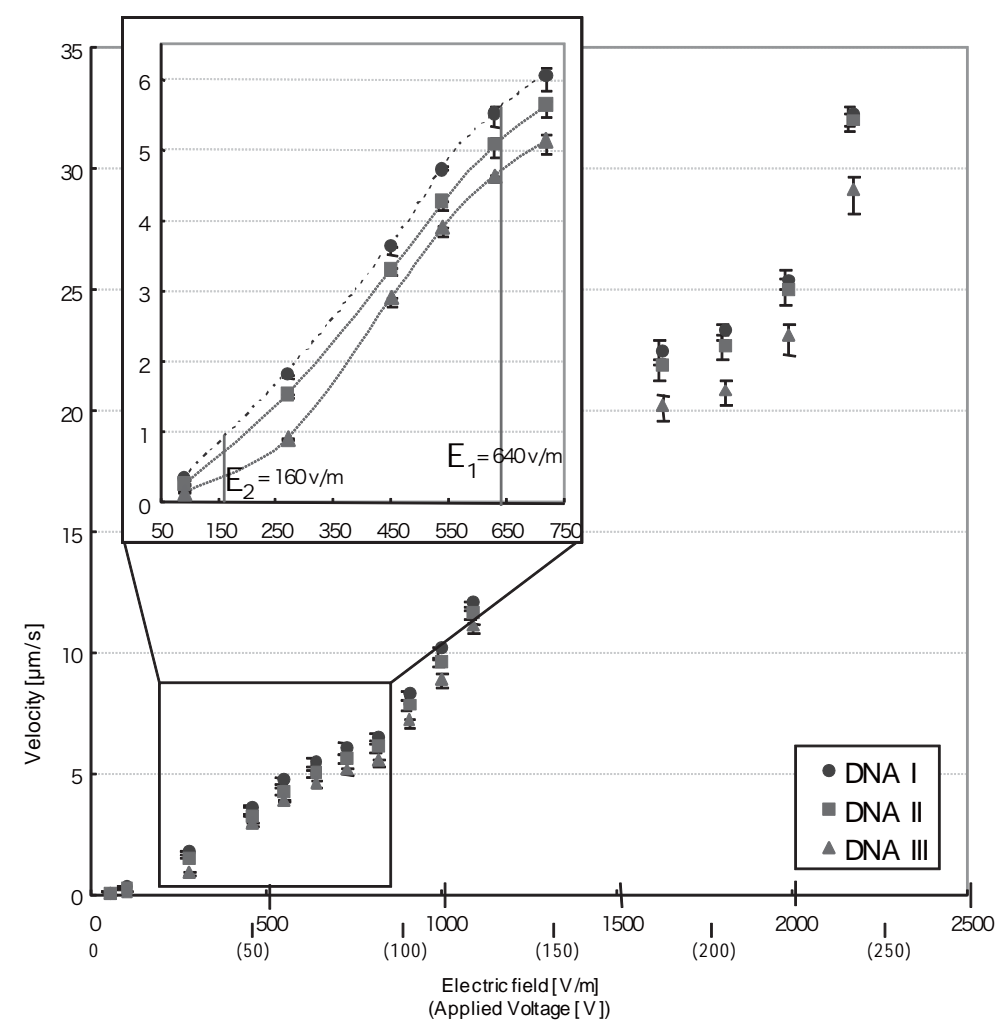

Fig. 3. Drift velocity of the three different DNA molecules, measured from the conventional agarose gel electrophoresis: $E_{1}$ and $E_{2}$ are the electrical field conditions for the zero net migration of DNA II at the time condition of $T_{1}: T_{2}=3: 23$. 
Fig. 1. The DNA separation chip has one channel that has a width variation to make the electric field gradient from $x=-L$ to $x=+L$. Since the electric field difference between the specific locations of DNA I and DNA II is less than between that of DNA II and DNA III, we varied the channel width gradient to shorten the distance between the specific locations of DNA II and DNA III; then, the electric field gradient between the specific locations of DNA II and DNA III is higher than between those of DNA I and DNA II as shown in Fig. 4(a). Through the Maxwell simulation of the designed DNA separation chip with the applied voltages of 40 and $-10 \mathrm{~V}$ across two electrodes, we could obtain the electric field intensity along the channel as shown in Fig. 4(b).

Using the measured DNA migration velocity graph (Fig. 3) and the electric field

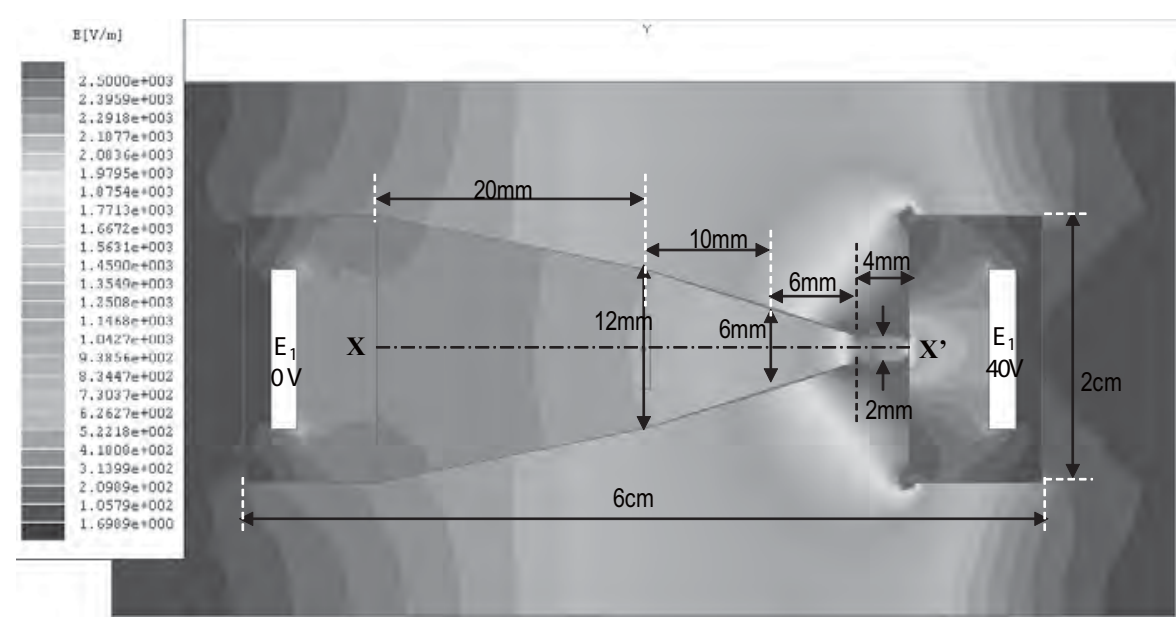

(a)

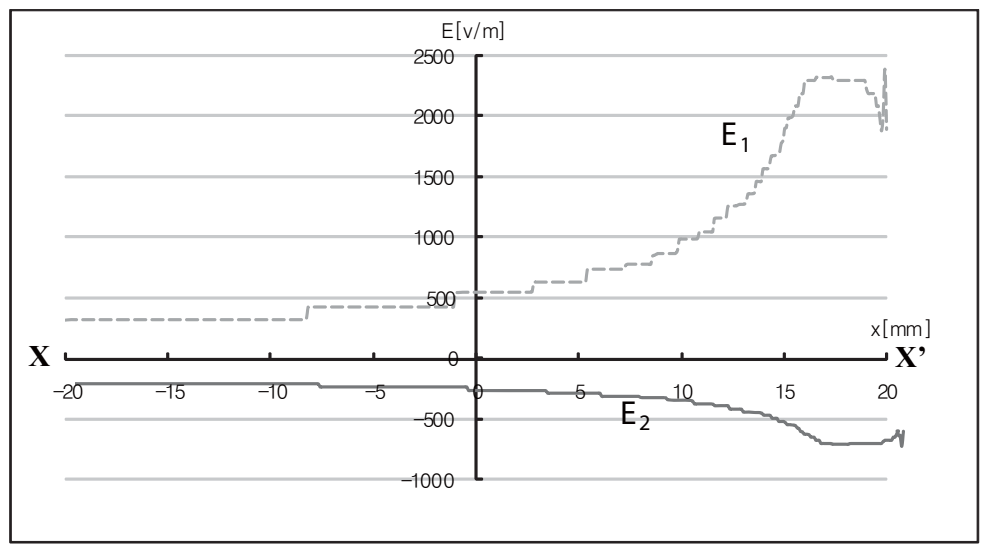

(b)

Fig. 4. Numerical analysis of the electric field in the DNA separation chip using agarose gel: (a) electric field distribution in the DNA separation chip, (b) electric field intensity according to $x$-axis along the line $\mathrm{X}-\mathrm{X}^{\prime}$ of (a). 
intensity(Fig. 4(b)), we estimated the migration of the sample DNA molecules along the channel under the asymmetric alternative electric field $\left(E_{1}\right.$ is $4 E_{2}$ and $23 T_{1}$ is $\left.3 T_{2}\right)$.

Figure 5 shows the simulated migration of the DNA molecules through the simulation program, which calculates the position of the DNA molecules every second using the velocity information with respect to the electric field values at the position of the DNA molecules in the channel. In the simulation, each DNA molecule migrated with a different net velocity according to its size; DNA I migrated backward and oscillated at $x=-1.02 \mathrm{~mm}$, DNA II and DNA III migrated forward and oscillated at $x=+2.30 \mathrm{~mm}$ and $x=+14.8 \mathrm{~mm}$, respectively, as shown in Fig. 6. Therefore, we can predict that all three sample DNA molecules could reach the specific locations where they show net zero migration velocity in 1200 min.

\subsection{Fabrication}

The fabrication of the DNA separation chip can be classified into three steps: fabrication of PDMS (PolyDiMethylSiloxane) channel, bonding the PDMS channel to the slide glass, and filling agarose gel in the PDMS channel. A channel for filling agarose gel was fabricated by the PDMS molding technique. A PDMS mixture (curing agent:PDMS $=1: 10$ ) was poured onto the molding jig and cured for $2 \mathrm{~h}$ at $85^{\circ} \mathrm{C}$. We defined the PDMS channel by cutting the PDMS replica. Before bonding a PDMS channel to the slide glass substrates, we treated the former with plasma generated by a high-frequency generator (BD-10AS, Electro-technic Products, Inc.) for the surface

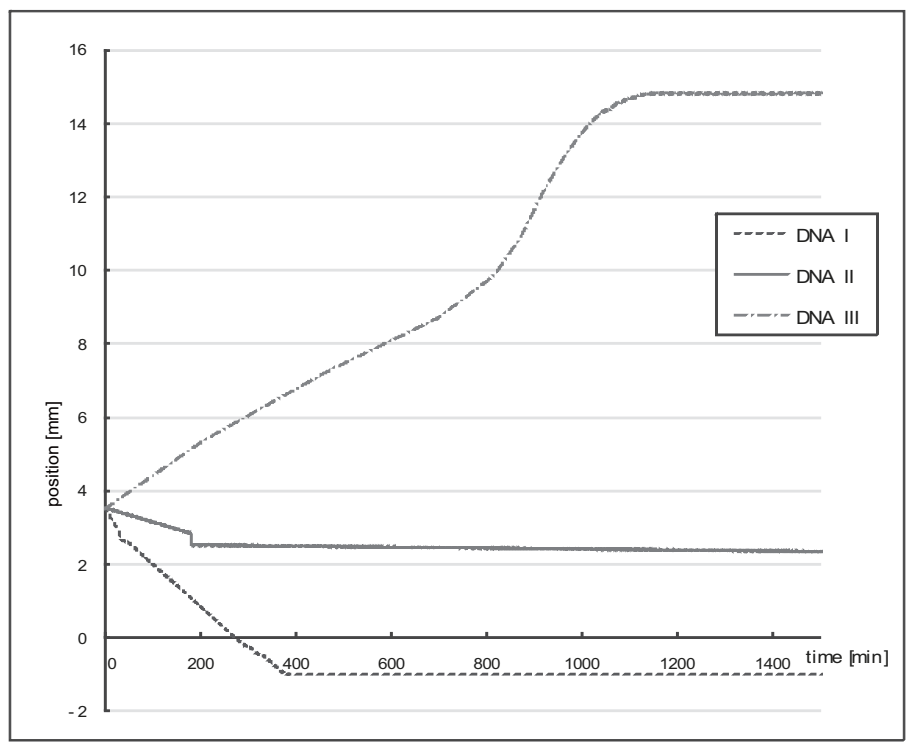

Fig. 5 Simulation of the migration of the sample DNA molecules under asymmetric alternating electric fields for estimating the specific locations where DNAs I (11.1 kbp), II (15.6 kbp), and III (48.5 $\mathrm{kbp}$ ) have net zero migration velocity. 


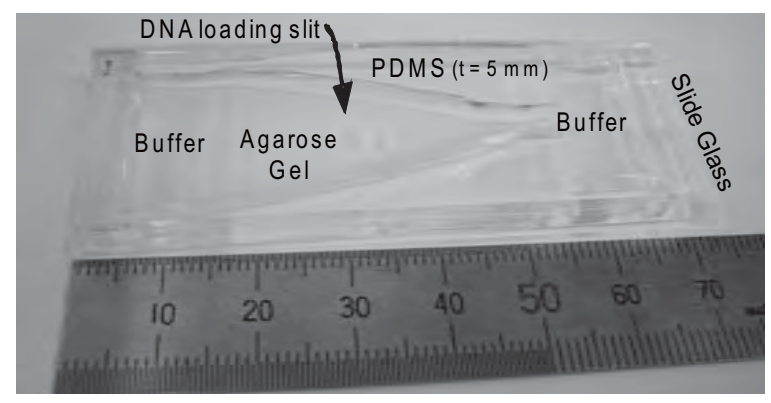

Fig. 6 Fabricated DNA separation chip.

activation. The plasma-treated PDMS channel and slide glass substrates were dipped in methanol for cleaning and bonding. After they were bonded, they were cured for $2 \mathrm{~h}$ at $120^{\circ} \mathrm{C}$. Finally, we filled the PDMS channel with the agarose I and TAE buffer mixture (agarose:buffer $=0.5 \mathrm{~g}: 100 \mathrm{ml}$ ). To form the congealed agarose gel in the channel, we boiled and filled the agarose I and buffer mixture in the PDMS channel, defining the slit for loading the DNA sample. Figure 6 shows the fabricated DNA separation chip filled with agarose gel.

\section{Experimental Results and Discussion}

In order to observe the DNA migration in the DNA separation chip, we used the test apparatus composed of UV lamp, camera, power supply, PC, and circuit for applying asymmetric alternating electric field train. Electric potential $(40 \mathrm{~V} /-10 \mathrm{~V})$ was applied through the platinum electrodes from the circuit. Through the $\mathrm{PC}$ using visual $\mathrm{C}++$ program, we regulated the period of the asymmetric alternating electric field train and regulated the amplitude of the electric field $E_{1}$ and $E_{2}$ through the 2 outputs of the power supply. Three kinds of DNA molecules were used to characterize the DNA separation chip. In order to detect the migration of the DNA molecules in the DNA separation chip on the UV-lamp, we stained the DNA molecules with the fluorescence dye, YOYO-1 (Molecular Probes Inc. Y-3601) at 5:1 ratio of base pairs to dye molecules. Since we used less DNA sample and the process time is longer compared with the conventional electrophoresis process, we stained the DNA molecules with YOYO-1 instead of EtBr.

When the DNA molecules migrated with the external electric field, we photographed the DNA migration at fixed time intervals using the CCD camera set above the DNA separation chip. Figure 7 shows the images captured from the fabricated DNA chip every $80 \mathrm{~min}$ of the separation process, demonstrating the size-dependent DNA separation in three different bands within the channel. In Fig. 8 showing the estimated and measured positions of each DNA molecule according to the process time, DNA II shows zero net migration velocity after $160 \mathrm{~min}$. DNA II migrated $1.5 \mathrm{~mm}$ backward from the initial point under the alternating asymmetric electric field, then reached the 
(a)

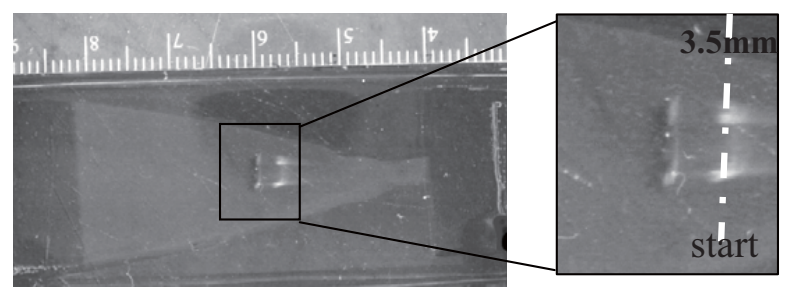

(b)

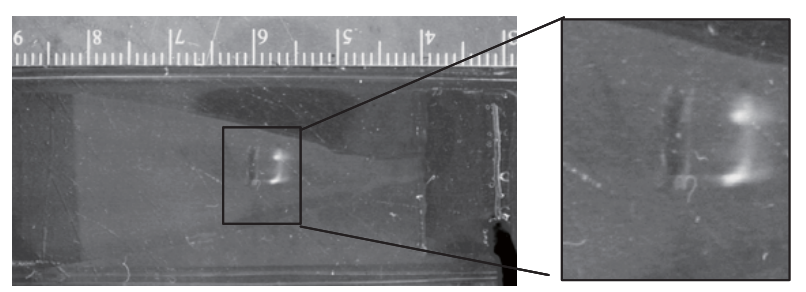

(c)

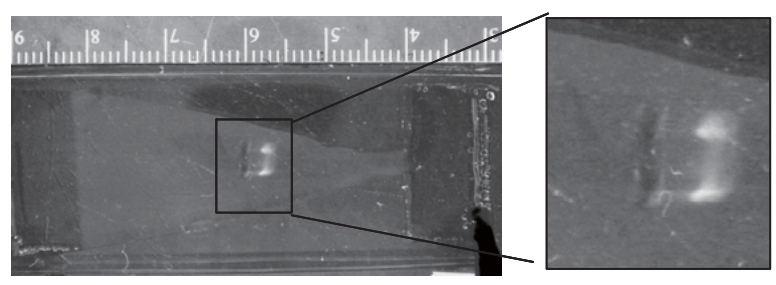

(d)

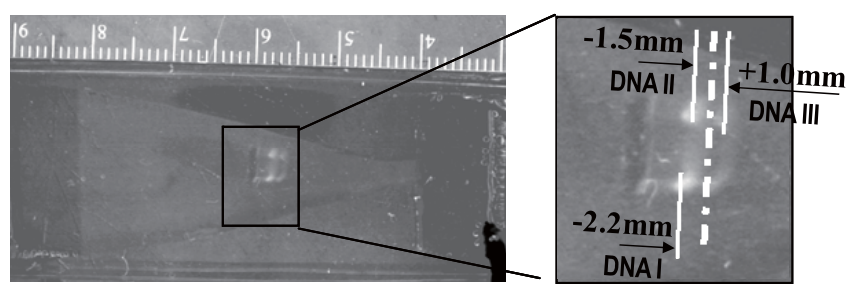

Fig. 7. Fluorescence images showing the separation of sample DNA molecules into the three different bands in the fabricated chip for the condition of Fig. 5: (a) initial image, (b) image after $80 \mathrm{~min}$, (c) image after $160 \mathrm{~min}$, (d) image after $240 \mathrm{~min}$. 


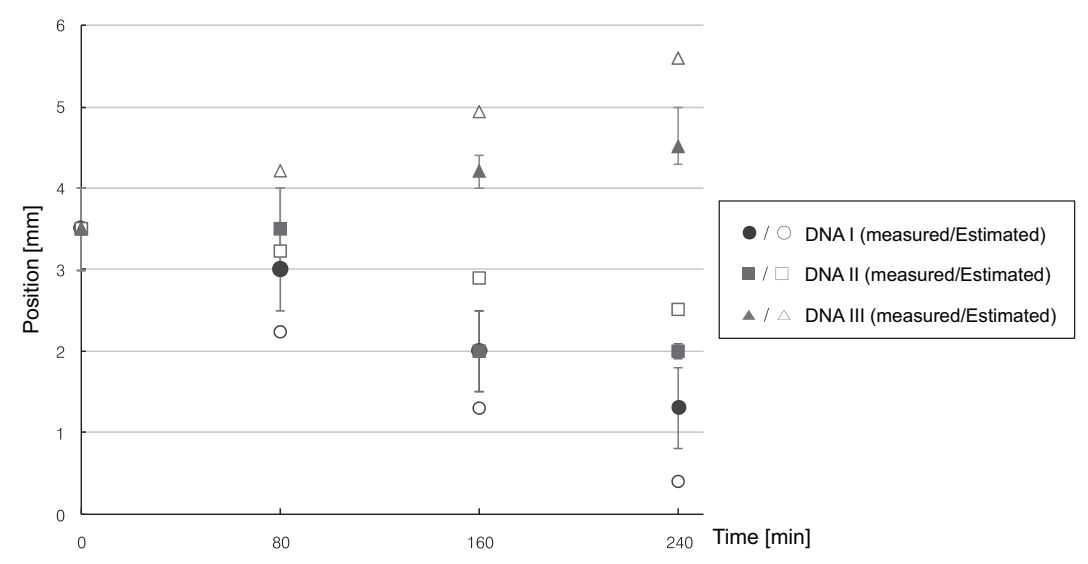

Fig. 8. Measured and estimated net migration distances for the three different DNA molecules.

point $2 \mathrm{~mm}$ from the DNA loading slit while showing net zero migration velocity. DNAs I and III, however, migrated $-2.2 \mathrm{~mm}$ (backward) and $+1.0 \mathrm{~mm}$ (forward), respectively, after $240 \mathrm{~min}$, toward their own specific locations. As estimated, each DNA molecule migrated to a different direction with a different net migration velocity according to its size under asymmetric alternating electric field train. DNA II reached the point $2 \mathrm{~mm}$ from $x=0$, while we estimated the specific location of DNA II $2.3 \mathrm{~mm}$ from the DNA loading slit. According to the measured specific location of DNA II, the actual applied electric field gradient is higher than the estimated one in Fig. 8. Since we manually placed an electrode in the DNA separation chip, the distance between 2 electrodes could be closer than in the model shown in Fig. 4. In the future, we should integrate electrodes on the substrate of the DNA separation chip to obtain a more stable result.

Although asymmetric alternating electric fields were applied for $240 \mathrm{~min}$, all the DNA molecules could not move to their own specific locations within the bleaching of the fluorescence dye, owing to an insufficient net migration velocity of the DNA molecules. To make all DNA molecules reach their own specific locations and have a zero net migration velocity, it is necessary to design a separation chip with a shorter channel to shorten the migration distance of the DNA molecules. The total time of DNA separation in the present chip (240 $\mathrm{min})$ is still longer than that of recently developed DNA separation chips using artificial gel structure (less than $1 \mathrm{~h}^{(4-6)}$ ). In this study, we were trying to verify the feasibility of DNA separation chip using the temporally asymmetric ratchet effect in nonuniform electric fields. By decreasing the separation chip sizes, we can decrease the total time of DNA separation time. If the channel shape is made hyperbolic, we could minimize the curvilinear shape of the separation tractions (a smiling effect). In addition, filling the sieving matrix that has more uniform pores can clarify the band of the DNA molecules in the channel. 


\section{Conclusions}

We present single-channel chips for ratcheting and separating DNA molecules using nonuniformly distributed asymmetric alternating electric fields in a PDMS microchip. We measured the drift velocities of three types of DNA molecules by varying the electric fields. On the basis of the drift velocity data, we found the proper conditions for the separation and trapping of DNA molecules and verified that DNA molecules migrate with the different velocities depending on their size under specific asymmetric alternating electric fields. Finally, one of the sample DNA molecules, DNA II, reached its own specific location, then showed zero net migration velocity, while DNA I and III migrated to the $-x$ and $+x$ directions, respectively. The proposed separation chips have potentials not only for the miniaturization of DNA analysis systems, but also for the tunable capability of the target DNA size to be separated and ratcheted by varying the amplitude and period of the asymmetric alternating electric fields and changing the gel property.

\section{Acknowledgements}

This work was supported by the National Creative Research Initiative Program of the Ministry of Science and Technology (MOST) and the Korea Science and Engineering Foundation (KOSEF) under the project title of "Realization of Bio-Inspired Digital Nanoactuators."

\section{References}

1 G. W. Slater, C. Desruisseaux, S. J. Hubert, J.-F. Mercier, J. Labrie, J. Boileau, F. Tessier and M. P. Pepin: Electrophoresis 21 (2000) 3873.

2 G. F. Carle, M. Frank and M. V. Olson: Science 232 (1986) 65

3 W. D. Volkmuth and R. H. Austin: Nature 358 (1992) 600.

4 T. A. J. Duke and R. H. Austin: Phys. Rev. Lett. 80 (1998) 1552.

5 L. R. Huang, J. O. Tegenfeldt, J. J. Kraeft, J. C. Sturm, R. H. Austin and E. C. Cox: Nat. Biotechnol. 20 (2002) 1048.

6 S. Y. Yi, K.-S. Seo and Y.-H. Cho: Sens. Actuators, A 120 (2005) 429.

7 J. Han, S. W. Turner and H. G. Craighead: Phys. Rev. Lett. 83 (1999) 1688.

8 J. Han and H. G. Craighead: Science 288 (2000) 1026.

9 T. A. J. Duke and R. H. Austin: Phys. Rev. Lett. 72 (1994) 2117.

10 L. L. Frumin, S. E. Peltek and G. V. Zilberstein: J. Biochem. Biophys. Methods 48 (2001) 269.

11 M. J. Chacron and G. W. Slater: Phys. Rev. E 56 (1997) 3446.

12 O. Lungu, T. C. Wright Jr., and S. Silverstein: Molecular and Cellular Probes 6 (1992) 145.

13 P. Kay, K. Meehan, and A. L. Williamson: J. Virol. Methods 105 (2002) 159.

14 M. Costa1, M. Dottori, K. Sourris, P. Jamshidi, T. Hatzistavrou, R. Davis, L. Azzola, S. Jackson, S. M. Lim, M. Pera, A. G. Elefanty and E. G. Stanley: Nature Protocols 2 (2007) 792.

15 O. Singer, G. Tiscornia, M. Ikawa and I. M Verma: Nature Protocols 1 (2006) 286.

16 H.P. Chou, C. Spence, A. Scherer and S. Quake: PNAS 96 (1999) 11. 